\title{
Retraction Note to: Complete Disjoint CoNP-Pairs but No Complete Total Polynomial Search Problems Relative to an Oracle
}

Titus Dose

\author{
Retraction Note to: \\ Chapter "Complete Disjoint CoNP-Pairs but No Complete \\ Total Polynomial Search Problems Relative to an Oracle" \\ in: L. A. Gąsieniec et al. (Eds.): Fundamentals \\ of Computation Theory, LNCS 11651, \\ https://doi.org/10.1007/978-3-030-25027-0_11
}

The author has retracted this chapter [1] because of a gap in the proof of the main theorem caused by an incorrect application of Claim 4. The author agrees to this retraction.

[1] Dose, T.: Complete disjoint CoNP-pairs but no complete total polynomial search problems relative to an Oracle. In: Gąsieniec, L., Jansson, J., Levcopoulos, C. (eds.) FCT 2019. LNCS, vol. 11651. Springer, Cham (2019). https://doi.org/10.1007/978-3030-25027-0_11. 